\title{
An Enhanced Smith Predictor Based Control System Using Feedback-feedforward Structure for Time-delay Processes
}

\author{
M. Mohammadzaheri ${ }^{a}$ and R. Tafreshi*b \\ a Department of Mechanical \& Industrial Engineering, College of Engineering, Sultan Qaboos University, Muscat, Oman. \\ ${ }^{\mathrm{b}}$ Reza Tafreshi, Mechanical Engineering, Texas A and M University at Qatar, PO Box 2387, Doha, Qatar.
}

Received 17 March 2017; Accepted 1 October 2017

\begin{abstract}
This paper presents enhanced Smith predictor based control systems (SPBCSs) for processes with a time-varying or fixed time delay. This work focuses on improving the arrangement and asynchrony of SPBCS components rather than the design of the predictor and the feedback controller, which have been well discussed in the literature. The proposed control system advances SPBCS through implementation of two design recommendations: (i) replacing the classical feedback controller by a feedback-feedforward control system, and (ii) synchronizing the reference signal and the predicted output. As a result, common shortcomings of SPBCSs or control systems based on Pade approximation, i.e. the trade-off between performance and steady-state error, and instability associated with non-minimum-phase systems do not exist in the proposed SPBCS. The superior performance of the proposed control system is demonstrated with two examples: temperature control of an infrared dryer (a system with fixed time-delay) and air-fuel ratio of a lean-burn spark-ignition engine (a system with time-varying delay and lag). The proposed control system is shown to clearly outperform the conventional SPBCS and Internal Model Control (IMC) PID based on Pade approximation for aforementioned examples and performs satisfactorily in the presence of noises, actuator saturations, and severe model inaccuracies.
\end{abstract}

Keywords: Smith predictor; Time delay system; Dryer; Engine.

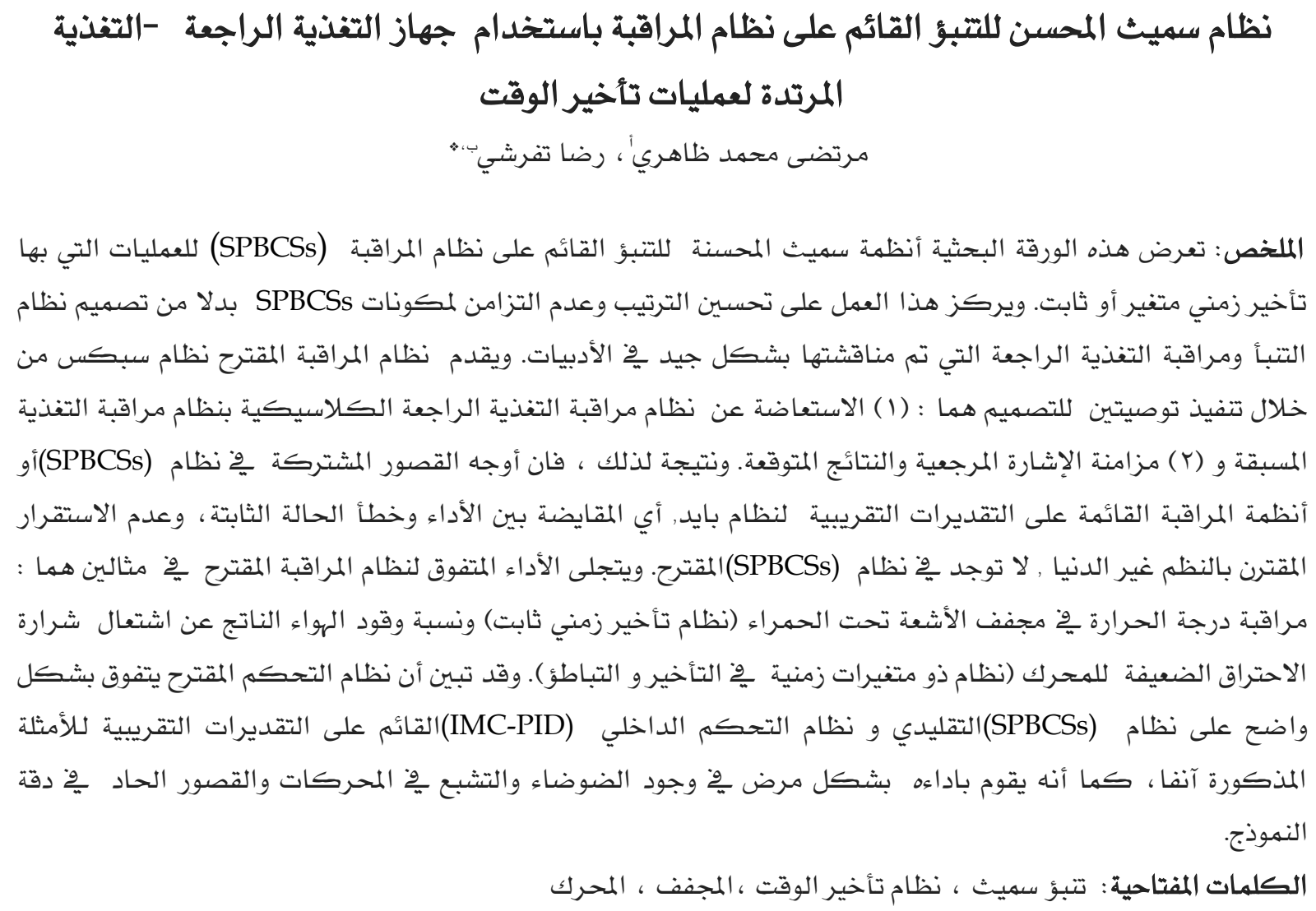

*Corresponding author's e-mail: Reza.tafreshi@qatar.tamu.edu 


\section{Introduction}

A wide variety of dynamic systems witness dead-time or delay. So called time-delay systems (Zhong 2006; Wu et al. 2010) include chemical processes, engines, manufacturing, micro-aerial vehicles and telecommunication systems, etc. (Liu 2014; Sanz et al. 2017). Timedelay is generally regarded as a main source of instability and poor performance in control systems ( $\mathrm{Li}$ and deSouza 1997). In order to effectively address this control issue, the difference between two categories of time-delay systems needs to be recognised. In the first category, states of a system influence their time derivative with a delay. This type of delay is mainly network-induced, i.e. it is resulted by the time needed to exchange data between devices in electrical/communication networks. The control of this type of time-delay is addressed using Lyapunov-Krasovskii and Razumikhin theorems (Wu et al. 2010) and is beyond the scope of process control and this paper. In the second category, the control inputs influence the time derivative of the system states with a delay, i.e. a noticeable time is needed for the actuator(s) to affect the states and the output (Zhong 2006). This type of time-delay is a prevalent concern in process control (Bequette 2008) and is studied in this paper.

Two main approaches have been employed to control the second category of time-delay systems: Pade approximation and predictive methods. In the first approach, the term presenting the delay is approximated using Pade technique (Bequette 2008). Then, the resultant model is used in control system design. Pade approximation results in model inaccuracy and transforms a stable minimumphase system to a non-minimum-phase one with a higher order (Ebrahimi et al. 2012). On the other hand, a predictive method, such as a Smith predictor (Smith 1957), does not add the aforementioned restrictions; hence, it is expected to present higher performance. Smith predictors were originally used for stable SISO systems, but further research extended their application to unstable systems (Kwak et al. 1999, Mataušek and Ribić 2012) and systems with long delays (Astrom et al. 1994). As a result, Smith predictors were suggested to be a part of control solution for any stable or unstable time-delay system (DePoar 1985; Zhong 2006).
Despite all the advantages and advances of SPBCSs, there are reports showing the superiority of feedback control systems based on Pade approximation over SPBCSs. For example, in the control of linear systems with identifiable time-varying delays (Ebrahimi et al. 2014a; Ebrahimi et al. 2014b; Mohammadzaheri et al. 2015a and 2015b). This research investigates and identifies two reasons behind relatively poor performance of SPBCSs and proposes two design recommendations to resolve the identified issues. Unlike most of the literature on SPBCSs, the focus of this research is on the structure of SPBCSs rather than the design of the predictor or the feedback controller. The proposed control system, an enhanced SPBCS, is assessed on systems with fixed or time-varying delays. A thorough assessment shows the superior performance of the proposed control system.

\section{Shortcomings of SPBCS}

Figure 1 shows a typical Smith-predictor-based control system (SPBCS), where $G(z)$ and $\hat{G}(z)$ are the plant original and approximated transfer functions without the delay, respectively, $r$ is the delay, and $C(z)$ is a linear classical controller designed for $G(z)$. Regardless of the methods used to design the predictor and the classical controller, this structure has two main shortcomings causing a relatively low performance:

(i) Design of a feedback classical controller (a vital part of SPBCS) is always a trade-off between performance and steady-state error. Moreover, windup phenomenon (the influence of actuator's saturation on integrals (Aryan et al. 2010)) needs to be compensated. These limits influence the performance of the SPBCS; where other methods, e.g. the ones based on Pade approximation, may use non-classical controllers (e.g. sliding mode (Ebrahimi et al. $2014 b)$ ) with no trade-off or windup issues.

(ii) Figure 2, a re-arrangement of Fig. 1, demonstrates that the reference $y_{d}(k)$ is actually compared with the estimated output at a different time, $\hat{y}(k+r)$, i.e. there is an asynchrony in conventional SPBCS. 


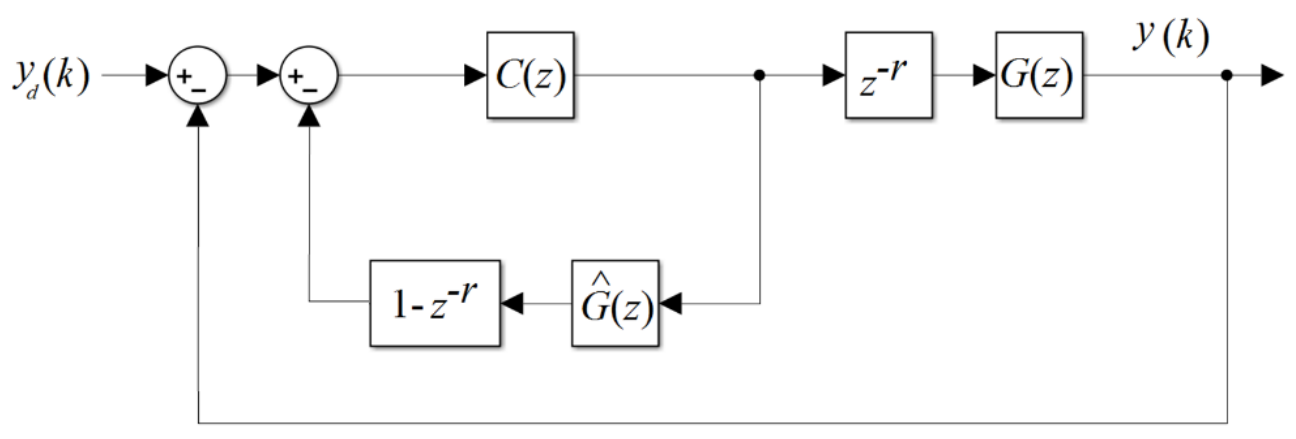

Figure 1. A conventional Smith-predictor-based control system.

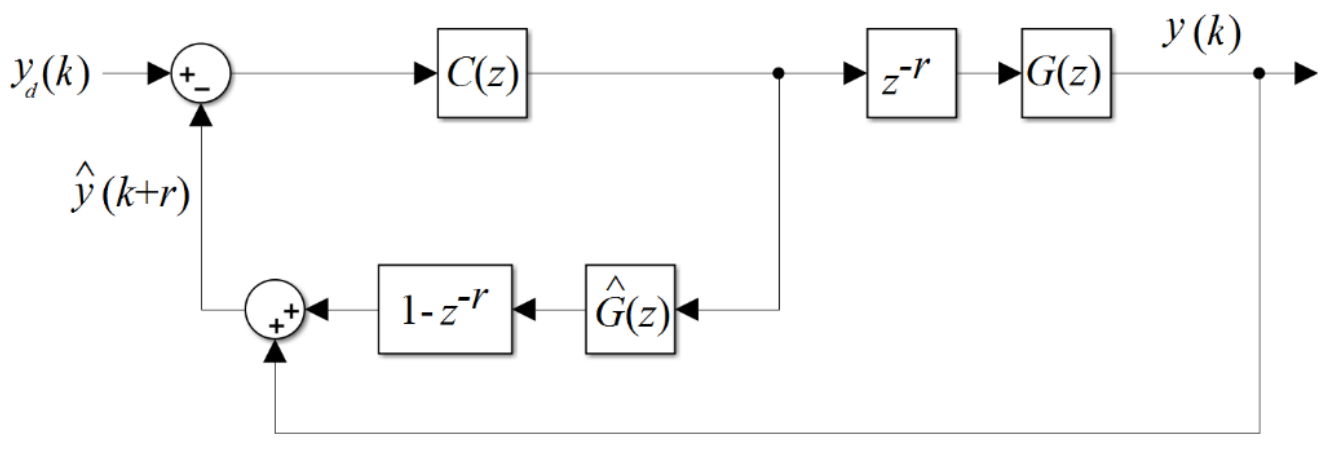

Figure 2. Another representation of the common Smith- predictor-based control system shown in Fig. 1 , assuming the discrepancy of $G(z)$ and $\hat{G}(z)$ is negligible.

Both aforementioned shortcomings are resolved in the proposed structure of SPBCS.

\section{Methodology}

The following design elements are recommended to address the shortcomings listed in the previous section.

Recommendation 1: A feedback-feedforward control law replaces the feedback controller of $C(z)$ in Fig. 2 (which is designed for the plant without delay or $G(z))$. The recommended control system is proposed in the following Corollary. This removes trade-off and windup issues.

Corollary: A process modelled by (1) or its discrete equivalent, is asymptotically stabilized by a control law of (2) if condition (3) is satisfied:

$$
G(s)=\frac{b}{T s+1},
$$

$u(t)=K e(t)+u^{*}(t)$,

$\frac{b K+1}{T}>0$,

where $e(t)=y_{d}(t)-y(t)$. Subscript $d$ refers to desired status $\left(y=y_{d}, \dot{y}=\dot{y}_{d}\right)$ and $u^{*}(t)$ is derived from (4):

$T \dot{y}_{d}(t)+y_{d}(t)=b u^{*}(t)$.

Proof is in the Appendix.

Accordingly, if, in (4), $b>0$ and $T>0$, with any positive $K$, stability is maintained, and steadystate error converges to zero. As a result, there is no trade-off between stability and steady-state error. Besides, there is no integral in (2); hence, windup does not exist. The sole source of performance restriction is the incapability to achieve high values of $K$ due to actuator limits. However, the feedforward controller, which generates $u^{*}(t), \quad$ is model-based. Hence, 
inaccuracy in model identification may lead to loss of performance as a disadvantage of the employed feedback-feedforward structure; this issue has been investigated for the proposed design in example 2. It should be noted that the control systems with feedforward-feedback structure have been investigated and used for different purposes (De Luca 2000; Bresch-Pietri and Krstic 2009; Mohammadzaheri et al. 2009). In this paper, this structure is adopted in SPBCSs.

Recommendation 2: In tracking problems, i.e. where the reference, $y_{d}$, is subject to change, the value of reference signal at $r$ instants ahead of current time, $y_{d}(k+r)$, is used both to generate feedforward control commands and to be compared with the output of the Smith predictor, as shown in Fig. 3. The value of reference signal is normally available upfront in process plants (Bresch-Pietri and Krstic 2009). This recommendation can be disregarded for regulation problems, where the reference is constant.

\subsection{Example1: An Infrared Dryer, A System with Fixed Delay}

Figure 4 shows an infrared dryer with a pair of lamps/thermocouples. Such dryers can be used to dry food and other bio products (Reed et al. 2000; Chuanzhu et al. 2005; Sharma et al. 2005; Roknul et al. 2014) or in a variety of other engineering applications (Mirsepahi et al. 2017). Using the experimental data and the least square method, the system transfer function has been approximated by (Mohammadzaheri et al. 2015a):

$$
\begin{gathered}
H_{\text {dryer }}(s)=\frac{52.7108}{1+152.812 s} e^{-1.3 s} \\
G_{c-\text { imc }- \text { dryer }}(s)=\frac{2.9114}{\lambda+0.65}+ \\
\left(\frac{0.0190}{\lambda+0.65}\right) \frac{1}{s}+\left(\frac{1.8844}{\lambda+0.65}\right)
\end{gathered}
$$

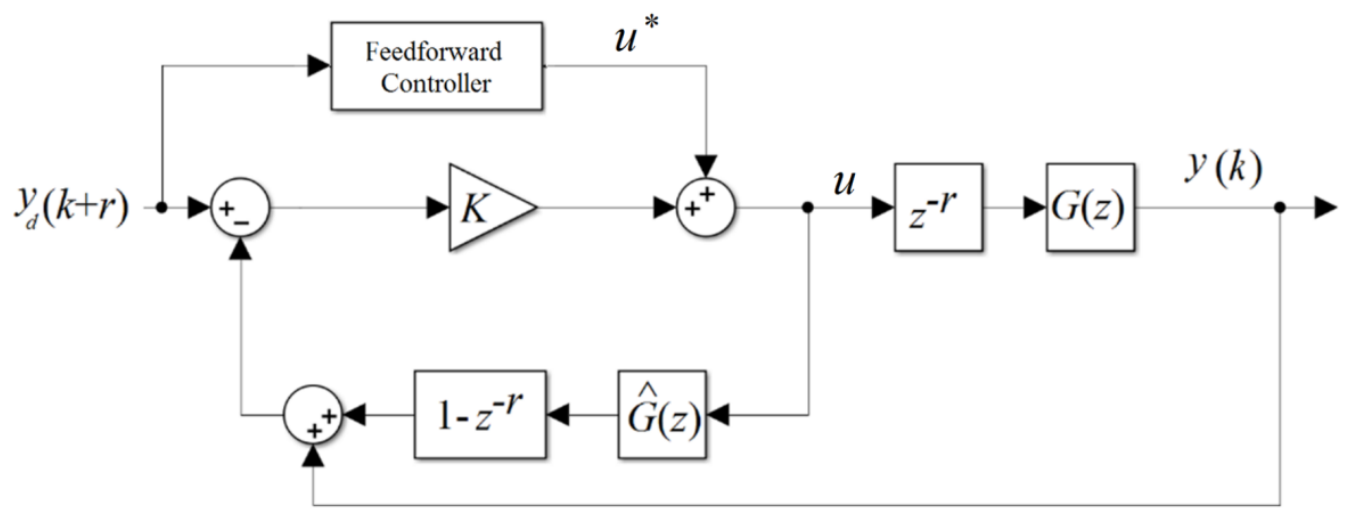

Figure 3. The proposed smith-predictor-based control system.

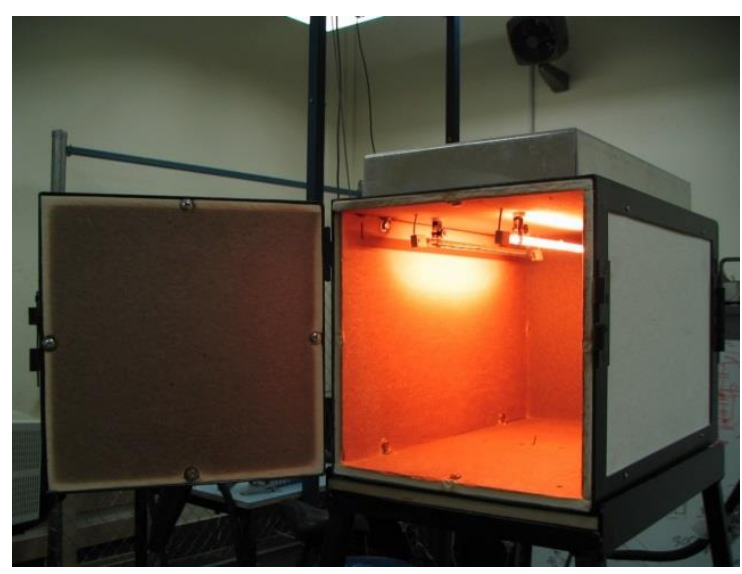

Figure 4 . The infrared dryer. 
The input is the control command (in the range of 0 and $10 \mathrm{~V}$ ) which excites the lamp power amplifier, and the output is the measured temperature in ${ }^{\circ} \mathrm{C}$. Equation (5) evidently meets the condition of the proposed corollary, i.e. (3), with any $K>0$.

Three control systems were examined on the dryer model:

(i) A feedback PID controller, (6), designed through Pade approximation and internal model control (IMC), with the method detailed in (Bequette 2008):

(ii) A conventional SPBCS (Fig. 1) with an IMC PI controller of (7) (a continuous equivalent of $C(z)$ in Fig. 1):

$$
C_{\text {dryer }}(s)=\frac{2.8991}{\lambda}+\frac{0.0190}{\lambda} \frac{1}{s},
$$

where $\lambda=1$ was chosen empirically for both (6) and (7).

(iii) The proposed enhanced SPBCS (Fig. 3) with feedforward component of $u^{*}=0.0190 y_{d}$, derived for the time-delay process presented by (5) at the steady state desired situation of $\dot{y}_{d}=0$. With such a feedforward component, the condition presented by (3) is satisfied for any positive feedback gain of $K$. A feedback gain of $K=1$ was chosen empirically to maintain reasonable trade-off between fast response and sensitivity to noise.

The mean of absolute controller error (MACE) was used as the control performance criterion:

$\mathrm{MACE}=\frac{\int_{t=t_{i}}^{t=t_{f}}\left|y_{d}(i)-y(i)\right| d t}{t_{f}-t_{i}}$.

where $t_{f}$ and $t_{i}$ are final and start times of operation. Figure 5 and Table 1 compare the performance of three controllers with and without a random noise in the range of $[-3+3]^{\circ} \mathrm{C}$ and with the fixed sampling frequency of 100 Hz. Feedback PID IMC fails to control the system in presence of noise. The proposed SPBCS outperforms both conventional SPBCS and PID IMC feedback with $9 \%$ to $22 \%$ less MACE.

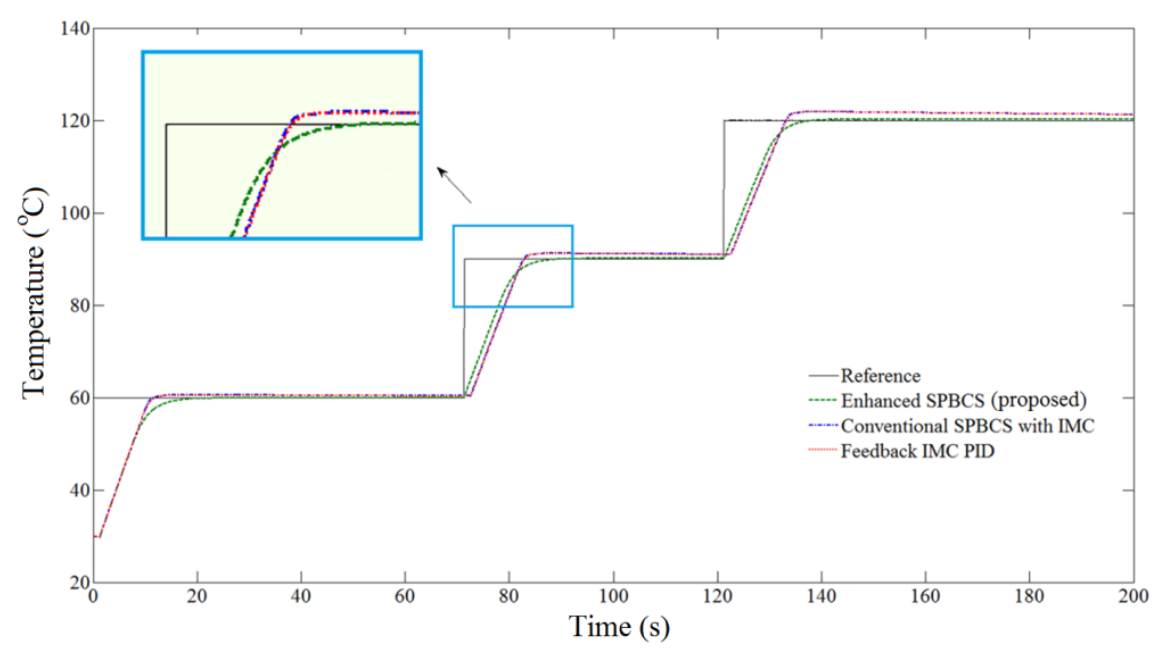

Figure 5. Performance of three control systems on an infrared dryer model without noise, where conventional IMC SPBCS and feedback IMC PID almost match.

Table 1. Mean of absolute control error for different controllers.

\begin{tabular}{lcc}
\hline & \multicolumn{2}{c}{ MACE [ $\left.{ }^{\circ} \mathrm{C}\right]$} \\
& Noise-less & With Noise \\
\hline IMC PID & 2.0068 & Failure \\
Conventional IMC SPBCS & 1.9911 & 2.2875 \\
Enhanced SPBCS & 1.5686 & 2.0898 \\
\hline
\end{tabular}




\subsection{Example 2 - Air to Fuel Ratio of a Lean-} Burn Engine, a System with TimeVarying Delay and Lag

The second example is control of normalized air-fuel ratio (AFR) of a lean-burn spark-ignition (SI) engine. The importance of this control problem is on rise due to increasing concerns and new regulations about emissions (Pace and Zhu 2014; Lin et al. 2017) and fuel consumption (Ghodsi et al. 2017). Normalized AFR is defined as following (Zope et al. 2011).

$$
A F R=\frac{1}{A F R_{s}} \frac{\dot{m}_{a}}{\dot{m}_{f}},
$$

where $\dot{m}_{a}$ and $\dot{m}_{f}$ are air and fuel mass flow rates, respectively, and $A F R_{s}$ is the stoichiometric air-fuel ratio which is commonly 14.7 for gasoline engines (Ebrahimi et al. 2012). Input $A F R$ to the engine can be calculated using measured fuel and air mass flow rates. The output AFR is measured by the universal exhaust gas oxygen (UEGO) sensor (Fig. 6). The input and output AFR relationship is approximated by a first-order transfer function (Zope et al. 2011).

$$
H_{\text {fuel path }}(s)=\frac{1}{T s+1} e^{-\theta s} .
$$

where lag $(T)$ and time delay $(\theta)$ can be estimated as following:
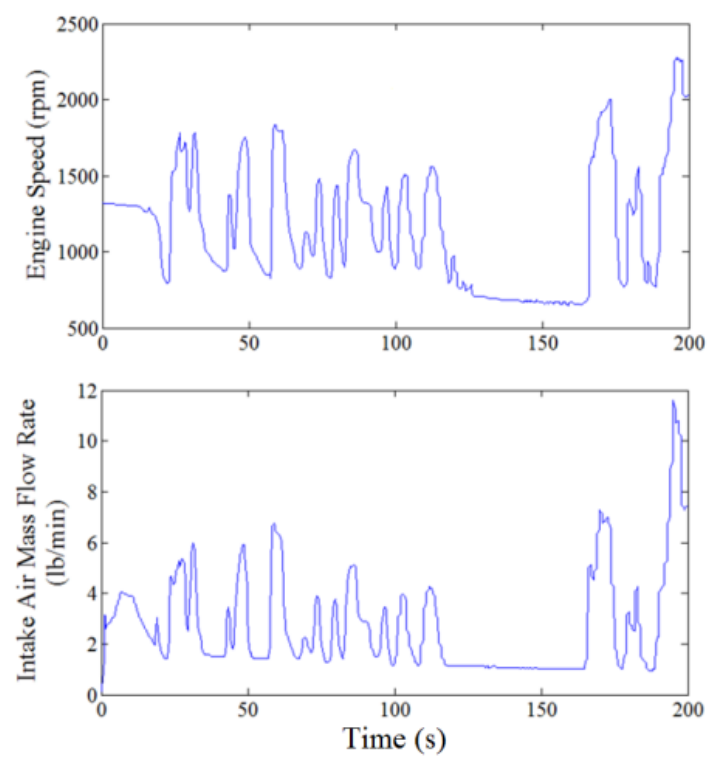

$\left\{\begin{array}{l}T=\frac{\alpha}{N}, \\ \theta=\frac{120}{N}+\frac{\beta}{\dot{m}_{a}},\end{array}\right.$

where $N$ is engine rotational speed, and $\alpha$ and $\beta$ are coefficients which are identified empirically with the values of $\alpha=997.4$ and $\beta=$ $0.0305 \mathrm{lb}$ for the investigated engine (Ebrahimi $e t$ al. 2012).

Figure 7 shows experimental data of engine speed and air mass flow as well as the time delay and lag time collected in a typical Federal Test Procedure (FTP) for an F-150 Ford truck with a V8 4.6L lean-burn SI engine (Ebrahimi et al. 2012).

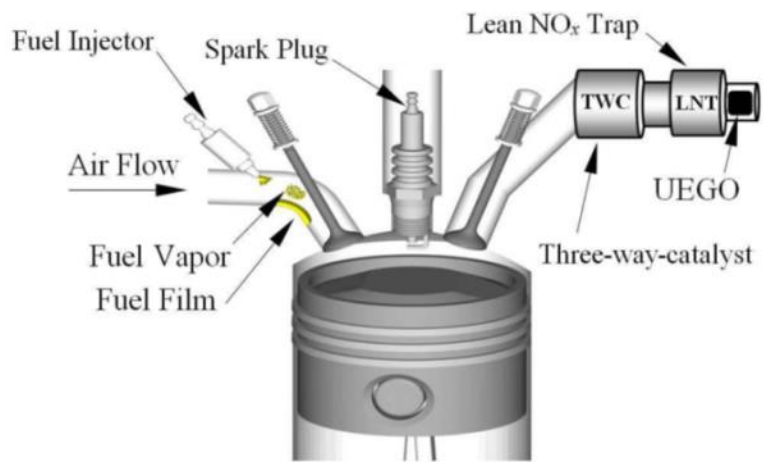

Figure 6. A schematic of air-fuel path in a leanburn SI engine (Ebrahimi et al. 2014).
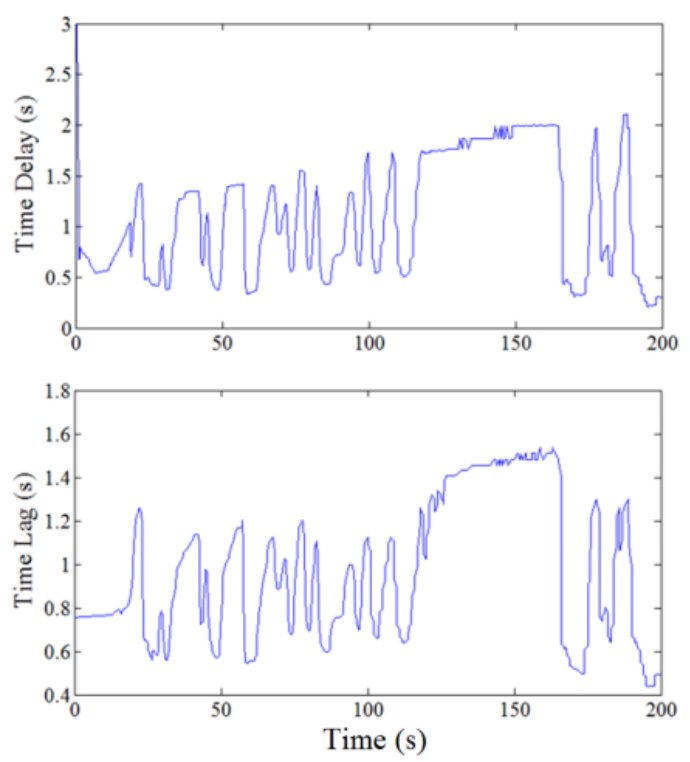

Figure 7. Engine speed and intake air flow mass rate according to a typical Federal Test Procedure (FTP) and time delay $(\theta)$ and lag time (T) calculated based on (11). 
For the aforementioned engine and operating conditions, depicted in Fig. 7, a feedback IMC PID was designed for (10). Furthermore, an IMC PI controller was designed for (10) excluding the exponential term to be used in the conventional Smith arrangement depicted in Fig. 1. For these controllers, the values of $\lambda$ were empirically found as 1.2 and 0.1, respectively. Equation (10) evidently meets the condition of the proposed corollary, i.e. (3), with any $K>0$. Therefore, the enhanced SPBCS (depicted in Fig. 3) is applicable on this system. The feedforward control command is $u^{*}=y_{d}$, based on ( 4 and 10). A feedback gain of $K=1$ was also empirically chosen. A random noise in the range of $\pm 0.5 \%$ of $A F R_{s}$ (Fiengo et al. 2005), i.e. \pm 0.0735 , was considered to assess the noise rejection of different control systems. A fixed sampling frequency of $12.5 \mathrm{~Hz}$, which is achievable by commercially available actuators (Woodward 2014) was also chosen. Figure 8 and Table 2 compare the performance of three investigated control systems with the control input saturation limits of 0 and 6 .

In this example, the coefficients of $\alpha$ and $\beta$ in (11) may be inaccurate due to imperfection of their identification process. In order to evaluate the effect of the inaccuracies, $20 \%, 30 \%$ and $50 \%$ of identification error were considered. Then, the control systems were designed based on inaccurate lag $(T)$ and delay $(\theta)$ obtained from the erroneous $\alpha$ and $\beta$ (Fig. 9). As demonstrated in Fig. 10 and Table 3, the proposed control system exhibits satisfactory performance with inaccurate $\alpha$ and $\beta$, where two other control systems witness either a significant drop in performance or total failure in most cases.

Table 2. Mean of absolute control error for different controllers on a lean-burn engine.

\begin{tabular}{lll}
\hline & \multicolumn{2}{c}{ MACE } \\
& Noise-less & With Noise \\
\hline Feedback IMC PID & 0.138 & 0.295 \\
Conventional IMC SPBCS & 0.122 & 0.286 \\
Enhanced SPBCS & 0.072 & 0.082 \\
\hline
\end{tabular}

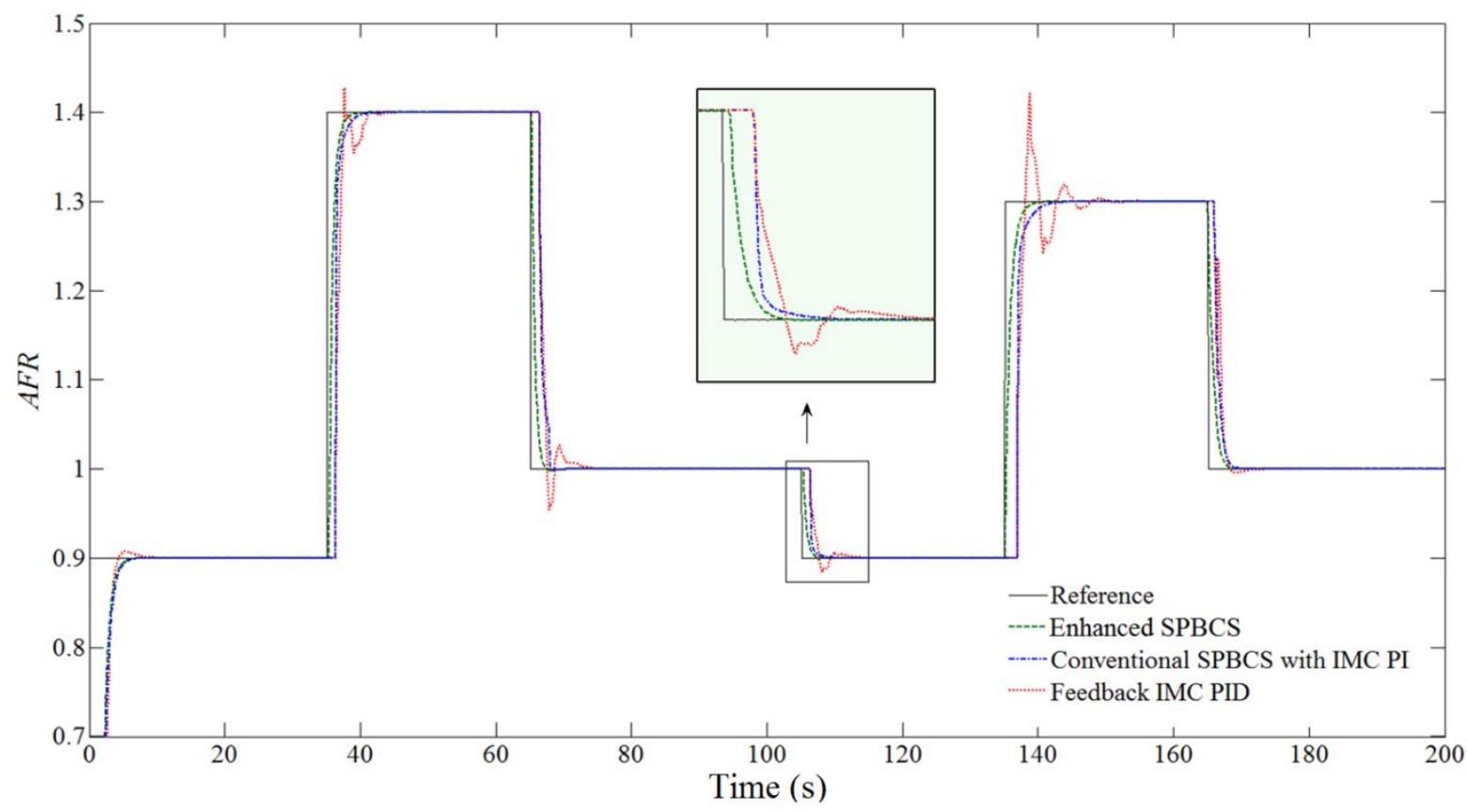

Figure 8. Performance of three control systems on an engine model without noise. 


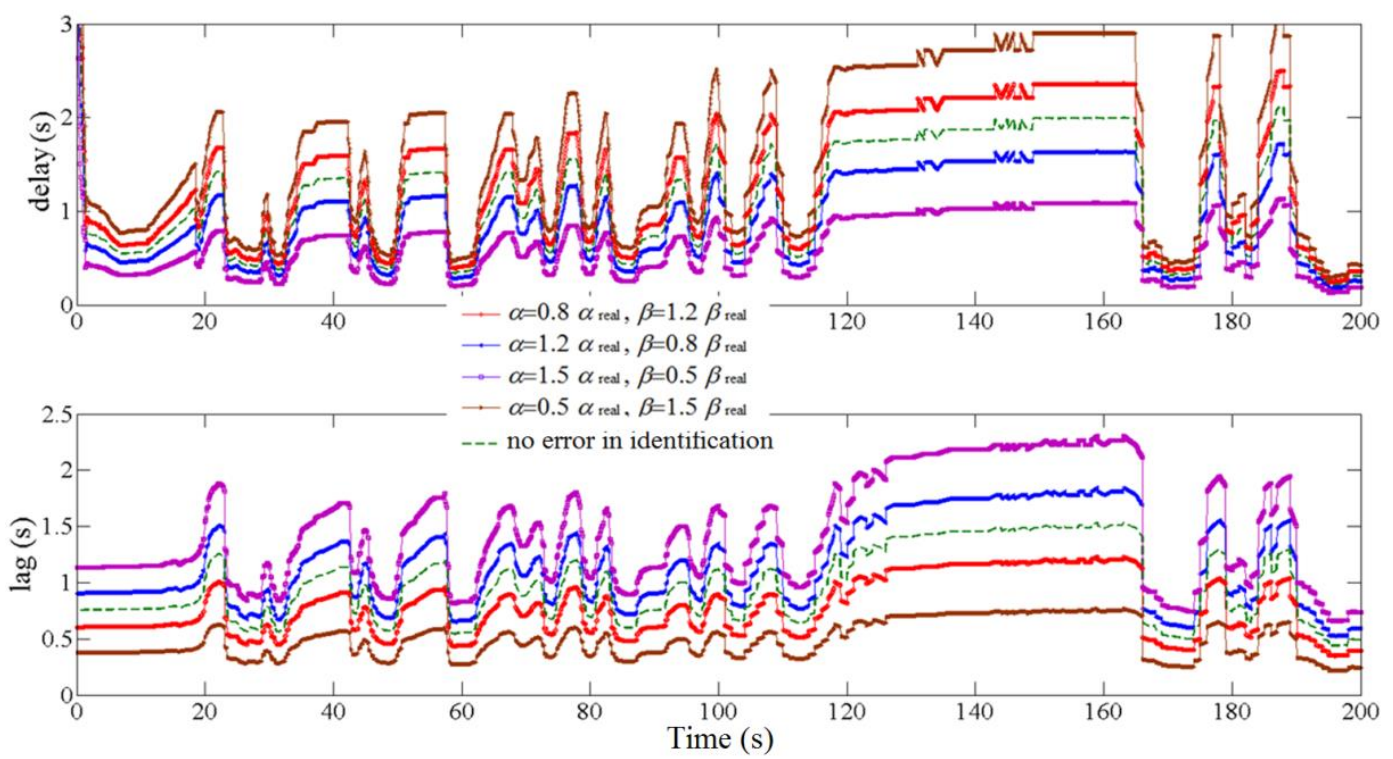

Figure 9. Delay and lag variations in the case of error in identification of $\alpha$ and $\beta$.

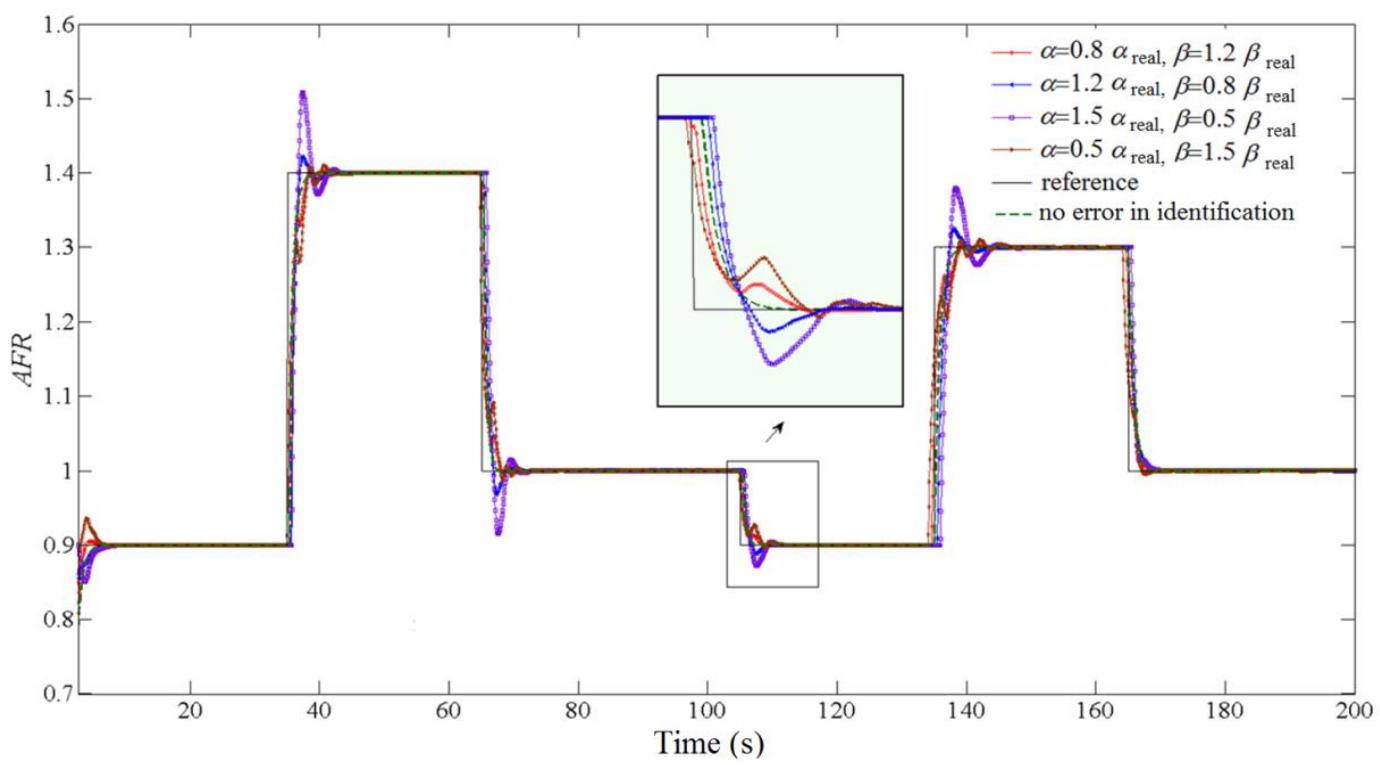

Figure 10. Performance of the enhanced SPBCS for AFR control in the case of error in identification of $\alpha$ and $\beta$.

Table 3. Mean of absolute control error with inaccurate parameters.

\begin{tabular}{lcccc}
\hline \multicolumn{1}{c}{ Control System } & \multicolumn{3}{c}{ MACE for various errors in identification of $\boldsymbol{\alpha}$ and $\boldsymbol{\beta}$} \\
& $\mathbf{+ 3 0 \% , \mathbf { 3 0 } \%}$ & $\mathbf{- 3 0 \% , + 3 0 \%}$ & $\mathbf{- 3 0 \% , - 3 0 \%}$ & $\mathbf{+ 3 0 \% , + 3 0 \%}$ \\
\hline Feedback IMC PID & 0.0147 & 0.0245 & Failure & Failure \\
Conventional IMC SPBCS & Failure & 0.0411 & 0.0582 & 0.1454 \\
Enhanced SPBCS & 0.0090 & 0.0076 & 0.0096 & 0.0076 \\
\hline
\end{tabular}

\section{Conclusion}

In this paper, two shortcomings of the structure of Smith predictor based control systems were identified: (i) inherited limits of the classical feedback controllers and (ii) asynchrony between the reference and the predictor output.
On this basis, two design recommendations were suggested to enhance SPBCSs: (i) replacing the feedback controller by a feedbackfeedforward structure and (ii) replacing reference signal at the current time by the reference signal at the target time of prediction. Within a corollary, the proposed control system 
was proved to produce a high performance with guaranteed stability for a wide class of timedelay processes. The enhanced SPBCS does not face issues of common control techniques for time-delay processes, i.e. windup, trade-off between performance and steady-state error, and non-minimum-phase systems (the latter is a result of Pade approximation). The proposed control system was assessed for processes with fixed/time-varying delays, in the presence of parameter identification errors and/or severe noises and/or actuator limits. The proposed control system exhibited superior performance in all cases and evidently outperformed a number of well-designed controllers based on Pade approximation or Smith predictor.

\section{Conflict of Interest}

The authors declare no conflicts of interest.

\section{Funding}

This work was supported by NPRP grant from the Qatar National Research Fund (a member of Qatar Foundation), grant number is 7-829-2-308.

\section{References}

Aryan P, Mohammadzaheri M, Chen L, Ghanbari M, Mirsepahi A (2010), GA-IMC based PID control design for an infrared dryer, Chemeca Adelaide, South Australia.

Astrom KJ, Hang CC, Lim B (1994), A new smith predictor for controlling a process with an integrator and long dead-time. IEEE transactions on Automatic Control 39(2): 343345 .

Bequette BW (2008), Process control, modeling, design and simulation. New Delhi, Prentice Hall of India.

Bresch-Pietri D, Krstic M (2009), Adaptive trajectory tracking despite unknown input delay and plant parameters. Automatica 45(9): 2074-2081.

Chuanzhu S, Xiangyou W, Chao G, Lifang P (2005), Development of far infrared dryer of $5 \mathrm{HY}$ model for vegetables [J]. Transactions of The Chinese Society of Agricultural Engineering 5: 020.

De Luca A (2000), Feedforward/feedback laws for the control of flexible robots, IEEE International Conference on Robotics and Automation San Francisco, USA.
DePoar A (1985), A modified smith predictor and controller for unstable processes with time delay. International Journal of Control 41(4): 1025-1036.

Ebrahimi B, Tafreshi R, Franchek M, Grigoriadis KM Mohammadpour J (2014a), A dynamic feedback control strategy for control loops with time-varying delay. International Journal of Control 87(5): 887-897.

Ebrahimi B, Tafreshi R, Masudi H, Franchek M, Mohammadpour J, Grigoriadis K (2012), A parameter-varying filtered PID strategy for air-fuel ratio control of spark ignition engines. Control Engineering Practice 20(8): 805-815.

Ebrahimi BR, Tafreshi J, Mohammadpour M, Franchek K, Grigoriadis, Masudi H (2014b), Second-order sliding mode strategy for air-fuel ratio control of lean-burn SI engines. IEEE Transactions on Control System Technology 22(4): 1374-1384.

Fiengo G, Grizzle J, Cook JA, Karnik AY (2005), Dual-UEGO active catalyst control for emissions reduction: Design and experimental validation. IEEE Transactions on Control Systems Technology 13(5): 722-736.

Ghodsi M, Hosseinzadeh N, Özer A, Dizaj HR, Hojjat Y, Varzeghani NG, Sheykholeslami MR, Talebian S, Ghodsi MH, Al-Yahmadi A (2017), Development of gasoline direct injector using giant magnetostrictive materials. IEEE Transactions on Industry Applications 53(1): 521-529.

Kwak HJ, Sung SW, Lee IB, Park JY (1999), A modified smith predictor with a new structure for unstable processes. Industrial and Engineering Chemistry Research 38(2): 405-411.

Li X, deSouza CE (1997), Delay-dependent robust stability and stabilization of uncertain linear delay systems: A linear matrix inequality approach. IEEE Transactions on Automatic Control 42(8): 11441148.

Lin JH, Chen P, Wang (2017), AFR control of a gasoline engine using triple-step method, 29th Chinese Control And Decision Conference, Chongqing, China.

Liu PL (2014), Further results on delay-rangedependent stability with additive timevarying delay systems. ISA Transactions 53(2): 258-266.

Mataušek MR, Ribić AI (2012), Control of stable, integrating and unstable processes by the modified smith predictor. Journal of Process Control 22(1): 338-343. 
Mirsepahi A, Mehdizadeh A, Chen L, O'Neill B, Mohammadzaheri M (2017), Comparison of inverse modelling and optimizationbased methods in the heat flux estimation problem of an irradiative dryer/furnace. Journal of Computational Science 19: 77-85.

Mohammadzaheri M, Chen L, Behnia-Willison F, Aryan P (2009), A design approach for feedback-feedforward control systems. IEEE International Conference on Control and Automation, Christchurch, New Zealand.

Mohammadzaheri M, Chen L, Mirsepahi A, Ghanbari M, Tafreshi R (2015), Neuropredictive control of an infrared dryer with a feedforward-feedback approach. Asian Journal of Control 17(5): 1972-1977.

Mohammadzaheri M, Tafreshi R, Ebrahimi B, Grigoriadis (2015), A predictive strategy to control time-varying delay systems: LeanBurn Engines, ASME Dynamic Systems and Control Conference, Columbus, Ohio, USA.

Pace S, Zhu GG (2014), Transient air-to-fuel ratio control of an spark ignited engine using linear quadratic tracking. Journal of Dynamic Systems, Measurement, and Control 136(2): 021008.

Reed TR, Meyer WJ, Van EM, Hermann D (2000), Infrared dryer system for printing presses, Google Patents.

Roknul AS, Zhang M, Mujumdar AS, Wang Y (2014), A comparative study of four drying methods on drying time and quality characteristics of stem lettuce slices (Lactuca sativa L.). Drying Technology 32(6): 657-666.

Sanz RP, García QC, Zhong, Albertos (2017), Predictor-based control of a class of timedelay systems and its application to quadrotors. IEEE Transactions on Industrial Electronics 64(1): 459-469.

Sharma G, Verma R, Pathare P (2005), Mathematical modeling of infrared radiation thin layer drying of onion slices. Journal of Food Engineering, 71(3): 282-286.

Smith OJ (1957), Closer control of loops with dead time. Chemical Engineering Progress 53(5): 217-219.

Woodward (2014), Fuel controls for engines. from http:// www.woodward.com / Applications-Engines.aspx.

Wu M, He Y, She JH (2010), Stability analysis and robust control of time-delay systems, Springer.

Zhong QC (2006), Robust control of time-delay systems, Springer.

Zope R, Mohammadpour J, Grigoriadis K, Franchek M, Tafreshi R, Masoudi H (2011), Identification of air-fuel ratio dynamics in SI engines using linear parameter varying techniques control and applications, Vancouver, Canada

\section{Appendix: Proof of the Corollary}

Eq. (1) results in $T \dot{y}(t)+y(t)=b u(t)$

Let us consider $\Delta u(t)=u^{*}(t)-u(t)$.

Subtracting (4) from (A.1) results in the error dynamics:

$T \dot{e}(t)+e(t)=b \Delta u(t)$.

With the control law of (2), $\Delta u(t)=-K e(t)$,

then $T \dot{e}(t)+e(t)=-b K e(t)$. Consequently, $\dot{e}(t)=-\left(\frac{b K+1}{T}\right) e(t) \Rightarrow e(t)=e\left(t_{0}\right) \exp \left[-\left(\frac{b K+1}{T}\right)\left(t-t_{0}\right)\right]$

As a result, if $\frac{b K+1}{T}>0$, the error consistently decreases and the closed loop system is asymptotically stable. 